\title{
INFINITE-BUS-EQUIVALENT ESTIMATION FROM LOCAL MEASUREMENTS ON A SYNCHRONOUS MACHINE
}

\author{
Alexandru Ţiclea and Gildas Besançon
}

\author{
Laboratoire d'Automatique de Grenoble \\ ENSIEG BP 46, 38402 Saint Martin d'Hères, France \\ fax: $+33(0) 476826388-$ tel: $+33(0) 476826230$ \\ e-mail: \{Alexandru.Ticlea,Gildas.Besancon\}@inpg.fr
}

\begin{abstract}
The present paper treats the rotor angle estimation problem in a singlemachine infinite bus setup. The components of the infinite bus voltage phasor are estimated in a coordinate frame that turns with the rotor by means of a Kalman-like filter from exclusively local measurements. Therefore, in addition to the rotor angle, the observer also provides an estimate of the infinite bus voltage. The proposed methodology is tested in simulation. Copyright (c)2005 IFAC
\end{abstract}

Keywords: Nonlinear systems, State estimation, Kalman filters, Power systems, Synchronous machines

\section{INTRODUCTION}

A power system is basically a collection of nonlinearly coupled systems - the generators - that supply electric power to some charges. Nonlinear behavior is not exclusively specific to generators; charges and some elements of the interconnecting network (e.g. FACTS devices) could also exhibit nonlinear dynamics. However, a power system is always required to operate at a specific point, which usually changes slowly on a future load prediction or planning basis. For this reason, relatively simple linear controllers were employed in practice in order to maintain transient stability and damp oscillations as far as small disturbances are concerned. As regards large disturbances, or those occurring when the system is loaded up to its stability limit, the performance of linear controllers can no longer match the performance of nonlinear ones.

This fact was recognized as soon as new developments in nonlinear control theory, such as linearization techniques, were applied to power systems. The first important result based on external feedback linearization (Marino, 1984), was further developed by several authors, for example Mak (1992), Mielczarski and Zajaczkowski (1994), King et al. (1994), Jain et al. (1994), Akhrif et al. (1999), Jiang et al. (2001). An alternative to this linearization technique is given by the direct feedback linearization, recent results being available for instance in (Guo et al., 2000; Guo et al., 2001). Somewhat better results can be obtained with the backstepping method, which in opposition to standard linearization techniques, preserves in closed loop the useful nonlinearities of the system. One of its applications to power systems can be found in (Roosta et al., 2001).

A common feature of these methods is that they use the state of the system in order to achieve the desired control action. One important state variable of a synchronous generator is the rotor angle, defined as the phase difference between the position of the rotor and a reference that turns at rated speed $\omega_{0}$. This reference could be the rotor of a specific generator in the power 
system, or a conveniently chosen voltage phasor. Unlike the internal rotor angle, defined as the phase difference between the position of the rotor and the terminal voltage phasor, which can be accurately computed from local measurements (de Mello, 1994), the rotor angle can only be computed if remote information is used.

Most authors admit indeed that the rotor angle is not readily available, but there are few solution proposals to overcome this problem. For instance, in (Mielczarski and Zajaczkowski, 1994) the authors propose an observer derived from the linearized model, while in (Wang et al., 1993), the angle is obtained during the transitory by simply integrating the rotor speed deviation with respect to $\omega_{0}$ under the assumption that the error upon the previous steady state value is within reasonable limits.

Since the vast majority of papers on nonlinear control in power systems involve a synchronous machine connected to an infinite bus (a concept that will be clarified in Section 2), the present paper proposes an observer that estimates the rotor angle along with the magnitude of the infinite bus voltage. The observer uses exclusively local measurements, namely the mechanical input torque, exciter output voltage and rotor speed deviation.

The transformations of the model that allow the design of such an observer are presented in Section 3. The corresponding simulation results are shown in Section 4 and then in Section 5 some conclusions are drawn.

\section{SINGLE-MACHINE INFINITE BUS SYSTEM MODEL}

As in the case of other electrical drives, the equations of the synchronous machine are written in a $\mathbf{d}-\mathbf{q}$ reference frame, which for this particular drive is chosen to revolve with the rotor. The complete set of equations and the significance of the $\mathbf{d}-\mathbf{q}$ coordinate system can be found for instance in (Kundur, 1994). Several degrees of simplification are possible, starting from the complete model, in order to obtain a model that reduces the computational effort in numerical simulations and preserves properties demanded by the analysis level. Generally, stator dynamics are neglected, such that interconnections between elements of the network are described through pure algebraical equations. At rotor side, the subtransient behavior, associated with the damper circuits, may be simplified or even eliminated from the model - see (Ilić and Zaborszky, 2000) for the most encountered approximations of the complete model. With the damper circuits neglected, one

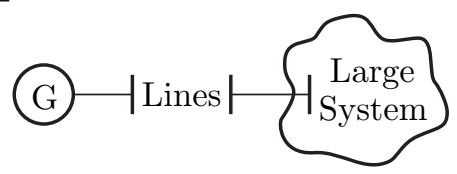

(a)

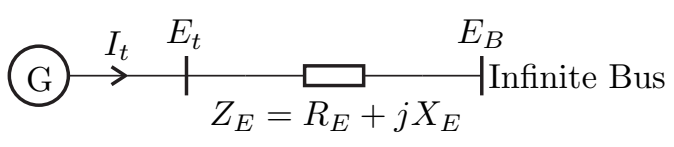

(b)

Fig. 1. Generator connected to a large system.

obtains the one-axis model, where the electrical characteristics of the machine are represented by a single differential equation, which describes the field circuit dynamics:

$$
\frac{\mathrm{d} E_{q}^{\prime}}{\mathrm{d} t}=\frac{\omega_{0}}{T_{d 0}^{\prime}}\left[E_{f d}-E_{q}^{\prime}-\left(X_{d}-X_{d}^{\prime}\right) i_{d}\right] .
$$

In the above equation, $E_{q}^{\prime}$ represents a voltage proportional to the field flux, $E_{f d}$ is the exciter output voltage, $X_{d}$ and $X_{d}^{\prime}$ represent the $\mathbf{d}$-axis synchronous and transient reactance respectively, $T_{d 0}^{\prime}$ represents the $\mathbf{d}$-axis open-circuit transient time constant and $i_{d}$ represents the $\mathbf{d}$-axis component of the stator terminal current.

The mechanical characteristics of the machine are described in terms of the rotor speed deviation $\Delta \omega_{r}$ with respect to the rated speed value $\omega_{0}$, and the angle $\delta$ between the position of the rotor and a reference that turns at rated speed:

$$
\begin{aligned}
\frac{\mathrm{d} \Delta \omega_{r}}{\mathrm{~d} t} & =\frac{1}{2 H}\left(T_{m}-T_{e}-K_{D} \Delta \omega_{r}\right) \\
\frac{\mathrm{d} \delta}{\mathrm{d} t} & =\omega_{0} \Delta \omega_{r},
\end{aligned}
$$

with

$$
T_{e}=E_{q}^{\prime} i_{q}+\left(X_{q}-X_{d}^{\prime}\right) i_{d} i_{q} .
$$

In these equations, $T_{m}$ and $T_{e}$ denote the mechanical and electromagnetic torque respectively, $H$ denotes the inertia constant, $K_{D}$ denotes the damping factor, $X_{q}$ denotes the q-axis synchronous reactance and $i_{q}$ denotes the $\mathbf{q}$-axis component of the stator terminal current.

Consider now a synchronous machine connected to a large power system through transmission lines. By means of Thévenin's theorem, the general setup of Figure 1(a) can be transformed in the form of Figure 1(b), where the transmission network and the external power system have been replaced by an equivalent impedance, $Z_{E}$, and an equivalent voltage, $E_{B}$. Since the machine is connected to a large system, it is reasonable to assume that its dynamics do not alter the voltage nor the frequency of the equivalent source $E_{B}$; it's the reason why this source is referred to as an infinite bus. However, the infinite bus voltage might change as a result of a change in the operating point of the external network. 


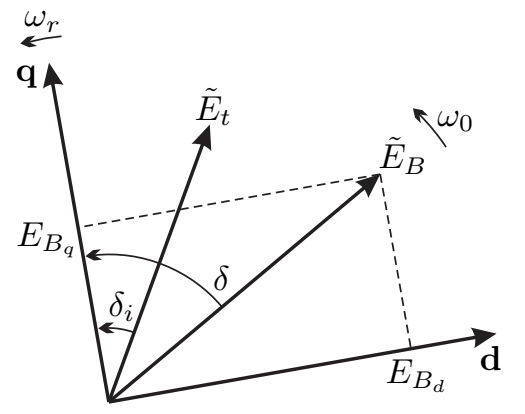

Fig. 2. Rotor angle illustration.

Under the assumption that the infinite bus voltage phasor $\tilde{E}_{B}$ always turns at speed $\omega_{0}$, it is natural to consider this phasor as the reference leaded by the $\mathbf{q}$-axis with the rotor angle $\delta$, as shown in Figure 2. The choice of the $\mathbf{q}$-axis as a means to identify the rotor position with respect to $\tilde{E}_{B}$ is due to the fact that the same axis is used to identify the internal rotor angle $\delta_{i}$ with respect to the stator terminal voltage phasor $\tilde{E}_{t}$. As a matter of fact, under no-load conditions, $\tilde{E}_{t}$ lies on the $\mathbf{q}$-axis, thus $\delta_{i}=0$, whereas under loaded conditions $\delta_{i}>0$.

Define now

$$
\begin{aligned}
& x_{d} \triangleq X_{d}+X_{E} \\
& x_{d}^{\prime} \triangleq X_{d}^{\prime}+X_{E} \\
& x_{q} \triangleq X_{q}+X_{E} .
\end{aligned}
$$

With stator and line resistances neglected, the components of the terminal current can be written for the setup of Figure 1(b) as

$$
\begin{aligned}
& i_{d}=\frac{E_{q}^{\prime}-E_{B_{q}}}{x_{d}^{\prime}} \\
& i_{q}=\frac{E_{B_{d}}}{x_{q}}
\end{aligned}
$$

with

$$
\begin{aligned}
& E_{B_{d}}=E_{B} \sin \delta \\
& E_{B_{q}}=E_{B} \cos \delta .
\end{aligned}
$$

Consequently, Equations (1) and (4) become respectively

$$
\frac{\mathrm{d} E_{q}^{\prime}}{\mathrm{d} t}=\frac{\omega_{0}}{T_{d 0}^{\prime}}\left(E_{f d}-\frac{x_{d}}{x_{d}^{\prime}} E_{q}^{\prime}+\frac{x_{d}-x_{d}^{\prime}}{x_{d}^{\prime}} E_{B_{q}}\right)
$$

and

$$
T_{e}=\frac{1}{x_{d}^{\prime}} E_{q}^{\prime} E_{B_{d}}-\frac{x_{q}-x_{d}^{\prime}}{x_{q} x_{d}^{\prime}} E_{B_{d}} E_{B_{q}} .
$$

Finally, taking the time derivatives of $E_{B_{d}}$ and $E_{B_{q}}$ and using Equation (3), one gets

$$
\begin{aligned}
\frac{\mathrm{d} E_{B_{d}}}{\mathrm{~d} t} & =\omega_{0} \Delta \omega_{r} E_{B_{q}} \\
\frac{\mathrm{d} E_{B_{q}}}{\mathrm{~d} t} & =-\omega_{0} \Delta \omega_{r} E_{B_{d}} .
\end{aligned}
$$

The differential equation of $\Delta \omega_{r}$ (2) along with Equations (9)-(12) completely describe the model of the generator connected to the infinite bus.

\section{BUS VOLTAGE AND ROTOR ANGLE ESTIMATION}

This section presents a solution for the estimation of $E_{B_{d}}$ and $E_{B_{q}}$ from available measurements of the output $\Delta \omega_{r}$ and inputs $T_{m}$ and $E_{f d}$.

The necessary step in order to design an observer for a nonlinear system is to put the system in a convenient form. Here, in a similar manner as in (Besançon and Ţiclea, 2003) for induction motors, the nonlinearities will be replaced by some auxiliary state variables, which will yield an affine representation with respect to the variables that are to be estimated.

The input-out map of the original system has to be preserved, so in general the construction starts by taking the output as the first state variable. Then, state variables are created conveniently so that an affine structure is obtained. The construction stops when the derivative of the last created variable only depends on already defined variables.

Starting from Equation (2), one solution is to replace $T_{e}$ by using (10) and then take $E_{q}^{\prime} E_{B_{d}}$ and $E_{B_{d}} E_{B_{q}}$ as state variables. An alternative is to directly take $T_{e}$ as state variable, which is the solution adopted herein. Both solutions yield in the end the same number of state variables, and apart from the possibility of estimating the electromagnetic torque directly, the second solution has no advantage over the first one.

By proceeding with the differentiation of $T_{e}$ with respect to time, one obtains

$$
\begin{aligned}
\frac{\mathrm{d} T_{e}}{\mathrm{~d} t}= & \Delta \omega_{r} \omega_{0}\left(a_{1} E_{q}^{\prime} E_{B_{q}}-a_{2} E_{B_{d}}^{2}+a_{2} E_{B_{q}}^{2}\right) \\
& +a_{3} E_{f d} E_{B_{d}}-a_{4} T_{e}+a_{5} E_{B_{d}} E_{B_{q}}
\end{aligned}
$$

with the following definitions of constants:

$$
\begin{aligned}
& a_{1} \triangleq \frac{1}{x_{d}^{\prime}} \\
& a_{2} \triangleq-\frac{x_{q}-x_{d}^{\prime}}{x_{q} x_{d}^{\prime}} \\
& a_{3} \triangleq a_{1} \frac{\omega_{0}}{T_{d 0}^{\prime}} \\
& a_{4} \triangleq \frac{\omega_{0}}{T_{d 0}^{\prime}} \frac{x_{d}}{x_{d}^{\prime}} \\
& a_{5} \triangleq a_{2} a_{4}+a_{3} \frac{x_{d}-x_{d}^{\prime}}{x_{d}^{\prime}} .
\end{aligned}
$$

In Equation (13), $E_{B_{d}}, E_{B_{d}} E_{B_{q}}, E_{B_{d}}^{2}, E_{B_{q}}^{2}$ and $E_{q}^{\prime} E_{B_{q}}$ have to be taken as state variables. The time derivative of $E_{B_{d}}(11)$ requires that $E_{B_{q}}$ be a state variable, for whom the time derivative is given by (12). As for the rest of variables, one has 


$$
\begin{aligned}
\frac{\mathrm{d} E_{B_{d}} E_{B_{q}}}{\mathrm{~d} t}= & \Delta \omega_{r} \omega_{0}\left(E_{B_{q}}^{2}-E_{B_{d}}^{2}\right) \\
\frac{\mathrm{d} E_{B_{d}}^{2}}{\mathrm{~d} t}= & 2 \Delta \omega_{r} \omega_{0} E_{B_{d}} E_{B_{q}} \\
\frac{\mathrm{d} E_{B_{q}}^{2}}{\mathrm{~d} t}= & -2 \Delta \omega_{r} \omega_{0} E_{B_{d}} E_{B_{q}} \\
\frac{\mathrm{d} E_{q}^{\prime} E_{B_{q}}}{\mathrm{~d} t}= & \Delta \omega_{r} \frac{\omega_{0}}{a_{1}}\left(T_{e}-a_{2} E_{B_{d}} E_{B_{q}}\right) \\
& -a_{4} E_{q}^{\prime} E_{B_{q}}+a_{6} E_{f d} E_{B_{q}} \\
& +a_{7} E_{B_{q}}^{2}
\end{aligned}
$$

where

$$
\begin{aligned}
& a_{6} \triangleq \frac{\omega_{0}}{T_{d 0}^{\prime}} \\
& a_{7} \triangleq a_{6} \frac{x_{d}-x_{d}^{\prime}}{x_{d}^{\prime}} .
\end{aligned}
$$

The set of equations $(11 \ldots 17)$ can be put in the form

$$
\begin{aligned}
\frac{\mathrm{d} x}{\mathrm{~d} t} & =A(u, y) x+B u \\
y & =C x,
\end{aligned}
$$

with

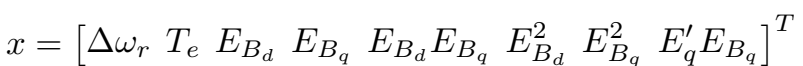

$$
\begin{aligned}
& u=\left[\begin{array}{ll}
T_{m} & E_{f d}
\end{array}\right]^{T} \quad y=\Delta \omega_{r}
\end{aligned}
$$

and the definitions of matrices $A, B$ and $C$ from Appendix A.

Further on, a Kalman-like observer can be designed as in Equations (20) and (21), which offers arbitrarily fast exponential convergence provided the existence of sufficient excitation (Besançon et al., 1996).

$$
\begin{aligned}
& \frac{\mathrm{d} \hat{x}}{\mathrm{~d} t}=A(u, y) \hat{x}+B u-S^{-1} C^{T}(C \hat{x}-y) \\
& \frac{\mathrm{d} S}{\mathrm{~d} t}=-\lambda S-A^{T}(u, y) S-S A(u, y)+C^{T} C
\end{aligned}
$$

\section{SIMULATION RESULTS}

The above estimation technique was tested in simulation for the system given by Equations (2), (3), (9) and (10) with the following values of parameters:

$$
\begin{gathered}
X_{d}=1.81 \mathrm{pu}, X_{d}^{\prime}=0.30 \mathrm{pu}, X_{q}=1.76 \mathrm{pu} \\
X_{E}=0.65 \mathrm{pu}, H=3.5 \mathrm{pu}, K_{D}=10 \mathrm{pu} \\
\omega_{0}=377 \mathrm{rad} / \mathrm{s}, T_{d 0}^{\prime}=8 \mathrm{~s}
\end{gathered}
$$

The initial values of the infinite bus voltage components $E_{B_{d}}$ and $E_{B_{q}}$ were such that at rated speed

$$
E_{t}=1 \mathrm{pu}, P=0.5 \mathrm{pu}, Q=0.3 \mathrm{pu},
$$

that is

$$
E_{B_{d}}=0.68 \mathrm{pu}, E_{B_{q}}=0.53 \mathrm{pu},
$$

or equivalently

$$
E_{B}=0.86 \mathrm{pu}, \delta=51.87 \text { degrees. }
$$

The observer was initialized with values computed from erroneous initial guesses of $E_{B_{d}}$ and $E_{B_{q}}$ that contained $50 \%$ and $100 \%$ errors respectively. The tuning parameter $\lambda$ was set at 500 .

With mechanical input torque and exciter output voltage kept constant at values corresponding to the above equilibrium point but with very low magnitude excitation added to the exciter output, the evolution of the estimation errors is presented by Figure 3 .

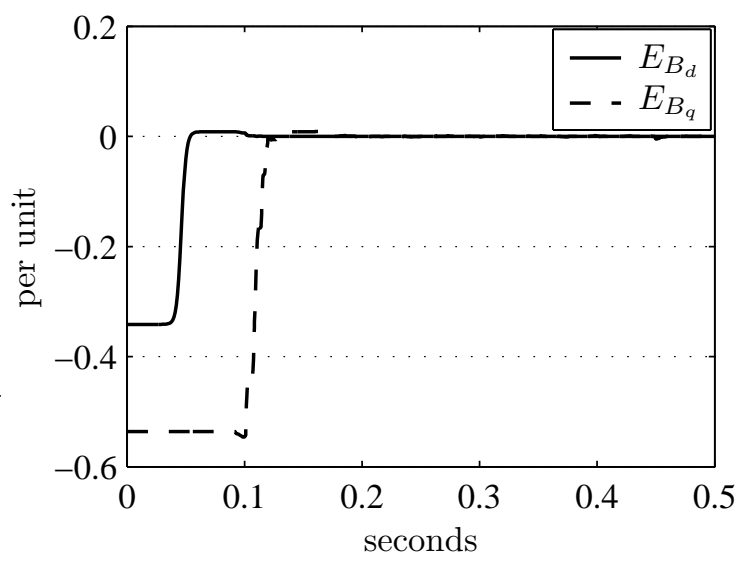

Fig. 3. Estimation errors at steady state.

With the system operating at the same point as above, a sudden increase of $0.04 \mathrm{pu}$ is now considered in the mechanical torque. As a result, the rotor oscillates until the new equilibrium is reached. Note that this kind of behavior is shared by another situation in which the mechanical power exceeds the electrical power, namely the sudden loss of a part of the load. For our example, the oscillations are illustrated in Figure 4 through the evolution of the infinite bus voltage components in rotor coordinates. The corresponding estimation errors are presented in Figure 5.

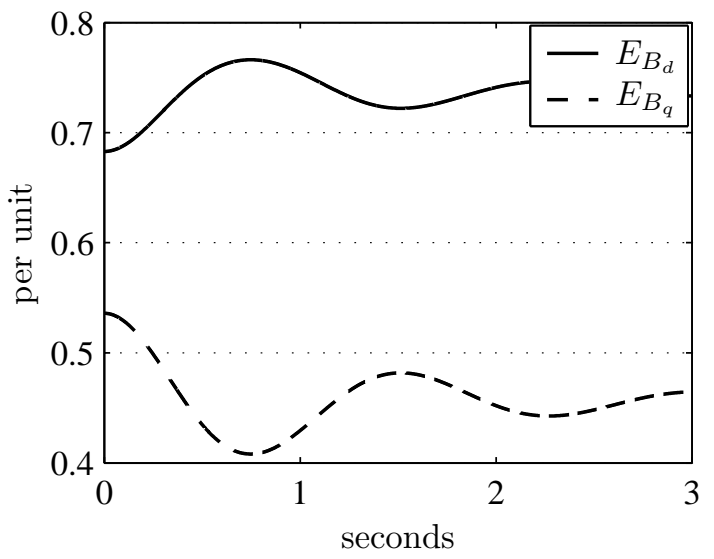

Fig. 4. Infinite bus voltage components in rotor coordinates during rotor swings. 


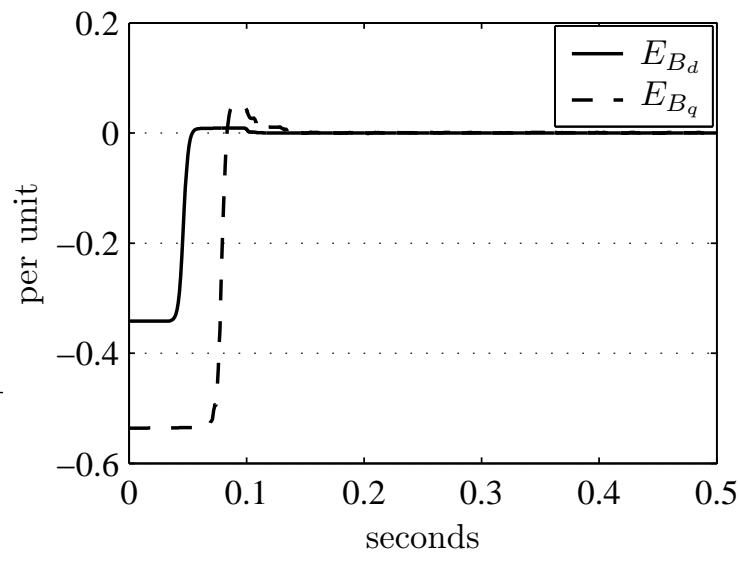

Fig. 5. Estimation errors during rotor swings.

\section{CONCLUSIONS}

The simulation results show that the proposed methodology is effective if sufficiently rich signals are supplied to the observer. Moreover, with this condition fulfilled the observer provides global exponential convergence, which is an important feature of the method.

Additional signals were added to the DC excitation voltage in order to ensure a sufficient frequency content, but it is worth noticing that in practice, the exciter's output is delivered by a commutation-based converter, which already provides a certain level of excitation. However, investigations through experiments on physical machines should check whether this is enough to ensure observer convergence.

From the power system point of view, the advantage is the possibility to estimate, in a configuration involving a generator and a large network, the magnitude of the infinite bus equivalent phasor and the angle between this phasor and the position of the machine's rotor. This information can be useful for both control and monitoring purposes.

Further improvements of this method, such as the estimation of line parameters and the validation of the estimator on more complex setups are the object of under way studies.

\section{REFERENCES}

Akhrif, O., F.-A. Okou, L.-A. Dessaint and R. Champagne (1999). Application of a multivariable feedback linearization scheme for rotor angle stability and voltage regulation of power systems. IEEE Transactions on Power Systems 14(2), 620-628.

Besançon, G. and A. Ţiclea (2003). Simultaneous state and parameter estimation in asynchronous motors under sensorless speed con- trol. Proceedings of the European Control Conference.

Besançon, G., G. Bornard and H. Hammouri (1996). Observer synthesis for a class of nonlinear control systems. European Journal of Control 2(3), 176-192.

de Mello, F. P. (1994). Measurement of synchronous machine rotor angle from analysis of zero sequence harmonic components of machine terminal voltage. IEEE Transactions on Power Delivery 9(1), 1770-1777.

Guo, G., Y. Wang and D. J. Hill (2000). Nonlinear output stabilization control for multimachine power systems. IEEE Transactions on Circuits and Systems 47(1), 46-53.

Guo, Y., D. J. Hill and Y. Wang (2001). Global transient stability and voltage regulation for power systems. IEEE Transactions on Power Systems 16(4), 678-688.

Ilić, M. and J. Zaborszky (2000). Dynamics and Control of Large Electric Power Systems. John Wiley \& Sons, Inc.

Jain, S., F. Khorrami and B. Fardanesh (1994). Adaptive nonlinear excitation control of power systems with unknown interconnections. IEEE Transactions on Control Systems Technology 2(4), 436-446.

Jiang, L., Q. H. Wu, J. Wang, C. Zhang and X. X. Zhou (2001). Robust observer-based nonlinear control of multimachine power systems. IEE Proceedings - Generation, Transmission and Distribution 148(6), 623-631.

King, C. A., J. W. Chapman and M. D. Ilić (1994). Feedback linearizing excitation control on a full-scale power system model. IEEE Transactions on Power Systems.

Kundur, P. (1994). Power System Stability and Control. McGraw-Hill, Inc.

Mak, F. K. (1992). Design of nonlinear generator exciters using differential geometric control theories. Proceedings of the Conference of Decision and Control, Tucson, AZ pp. 11491153.

Marino, R. (1984). An example of nonlinear regulator. IEEE Transactions on Automatic Control 29, 276-279.

Mielczarski, W. and A. M. Zajaczkowski (1994). Nonlinear field voltage control of a synchronous generator using feedback linearization. Automatica 30(10), 1625-1630.

Roosta, A. R., D. Georges and N. Hadj-Said (2001). Nonlinear control for power systems based on a backstepping method. Proceedings of the Conference of Decision and Control, Orlando, FL.

Wang, Y., D. J. Hill, L. Gao and R. H. Middleton (1993). Transient stability enhancement and voltage regulation of power systems. IEEE Transactions on Power Systems 8(2), 620627. 
Appendix A. DEFINITION OF MATRICES IN

EQUATIONS (18)-(19)

$$
\begin{aligned}
& A=\left[\begin{array}{cccccccc}
-\frac{K_{D}}{2 H} & -\frac{1}{2 H} & 0 & 0 & 0 & 0 & 0 & 0 \\
0 & -a_{4} & a_{3} u(2) & 0 & a_{5} & -a_{2} \omega_{0} y & a_{2} \omega_{0} y & a_{1} \omega_{0} y \\
0 & 0 & 0 & \omega_{0} y & 0 & 0 & 0 & 0 \\
0 & 0 & -\omega_{0} y & 0 & 0 & 0 & 0 & 0 \\
0 & 0 & 0 & 0 & 0 & -\omega_{0} y & \omega_{0} y & 0 \\
0 & 0 & 0 & 0 & 2 \omega_{0} y & 0 & 0 & 0 \\
0 & 0 & 0 & 0 & -2 \omega_{0} y & 0 & 0 & 0 \\
0 & \frac{\omega_{0}}{a_{1}} y & 0 & \omega_{0} u(2) & -\frac{a_{2}}{a_{1}} \omega_{0} y & 0 & a_{7} & -a_{4}
\end{array}\right] \\
& B=\left[\begin{array}{cc}
\frac{1}{2 H} & 0 \\
0 & 0 \\
\ldots \ldots & \ldots \\
0 & 0
\end{array}\right] \\
& C=\left[\begin{array}{llll}
1 & 0 & \cdots & 0
\end{array}\right]
\end{aligned}
$$

